\title{
Philosophiques
}

\section{Raymond Klibanski et David Pears (dirs), La philosophie en Europe, Paris, Gallimard/Unesco, 1993, 815 pages.}

\section{René O. Girard}

Volume 23, numéro 1, printemps 1996

Critères esthétiques et métamorphoses du beau

URI : https://id.erudit.org/iderudit/027384ar

DOI : https://doi.org/10.7202/027384ar

Aller au sommaire du numéro

Éditeur(s)

Société de philosophie du Québec

ISSN

0316-2923 (imprimé)

1492-1391 (numérique)

Découvrir la revue

Citer ce compte rendu

Girard, R. O. (1996). Compte rendu de [Raymond Klibanski et David Pears (dirs), La philosophie en Europe, Paris, Gallimard/Unesco, 1993, 815 pages.]

Philosophiques, 23(1), 188-190. https://doi.org/10.7202/027384ar d'utilisation que vous pouvez consulter en ligne.

https://apropos.erudit.org/fr/usagers/politique-dutilisation/ 
Raymond Klibanski et David Pears (dirs), La philosophie en Europe, Paris, Gallimard/Unesco, 1993, 815 pages.

Ce livre est un collectif qui passe en revue les principaux courants philosophiques en Europe. Un tel projet a l'avantage de démystifier la vision réductrice des universités nord-américaines sur la philosophie en Europe qu'elles désignent sous le vocable de "philosophie continentale *. C'est le projet en tout cas qu'ont voulu mener Raymond Klibansky et David Pears, présidents honoraires de l'Institut international de philosophie, avec la collaboration de la Division de philosophie et d'éthique (DPE) de l'UNESCO.

Ce livre comporte deux sections dont les chapitres sont autant de contributions d'auteurs différents. La première section, intituleee "Pays ", consiste en un vaste éventail des principaux courants philosophiques de chacun des pays européens, à l'exception de (feu) la Yougoslavie. Certains auteurs se contentent de montrer, de façon très succincte, les tendances spécifiques aux régions étudiées; c'est le cas de Rudolf Haller pour l'Autriche où l'on découvre un renforcement des tendances empiristes et analytiques; Paul Gochet montre l'importance de la logique et de la philosophie des sciences en Belgique ; Anani Stojnev montre qu'en Bulgarie la philosophie des quinze dernières années est passée de l'abstrait au concret en s'engageant dans l'êtude des problèmes de la vie courante : avec Evanghelos Mitsopoulos, on remarque que la Grèce est encore fortement influencée par le platonisme dont la marque s'impose à toutes les nouvelles tendances que l'auteur énumère ; la philosophie en Hongrie est présentée sous deux aspects : d'une part elle est évaluée dans son rapport avec la politique du pays par Attila Beckskehazi et Imre Marton, d'autre part elle fait l'objet d'une mise en situation des divers courants qui circulent au pays par Ferenc L. Lendvai : Gabriel Nuchelmans expose brievement l'organisation de l'enseignement de la philosophie au niveau universitaire et signale une certaine tendance à l'éclectisme pour une bonne partie de la philosophie néerlandaise : Alain Guy pour l'Espagne et le Portugal, Alexandre Tanase pour la Roumanie, $\mathrm{T}$. A. Alekseeva pour la Russie, Gabrielle-Dufour-Kowalska pour la Suisse romande, Henri Lauener pour la Suisse alémanique, Petr Horák et Martin Profant pour la Tchécoslovaquie, enfin Ioanna Kuçuradi pour la Turquie montrent les variations et les accents passés et actuels concernant le champ philosophique.

D'autres auteurs développent et insistent un peu plus longuement sur certains aspects spécifiques comme c'est le cas pour Reiner Wiehl pour l'Allemagne. Ce dernier fait remarquer le regain d'intérêt pour le néokantisme que la période du national-socialisme avait relégué aux oubliettes. La philosophie de Cassirer est très en vogue, elle " jette un pont audacieux entre les questions de l'idéalisme kantien et la philosophie du langage de Humboldt, d'une part, et les questions de sémiotique, d'analyse des formes mythiques, d'autre part, ainsi que les questions relatives à la fondation d'une anthropologie universelle des cultures"(p. 63). La philosophie analytique a connu aussi de nouveaux développements dans la philosophie de langue allemande surtout grâce à linfluence de Wittgenstein.

En France, on assiste à un regain d'inquiètude èthique et à un renouveau d'intérèt pour la question des droits de l'homme. Dominique Janicaud montre qu'en France, malgré un chapelet d'œuvres monadiques, des lignes de force se dégagent et se ramènent à ce que l'auteur appelle une nouvelle intelligibilité philosophique, appelée "rationalité plurielle " (p. 159). Cette rationalité se situe au croisement de nombreuses tendances, qui illuminèrent le ciel français d'abord avant d'étre exporté outre-Atlantique comme ce fut le cas pour 
l'existentialisme et pour le déconstructivisme derridien qui scintillent encore, bien que faiblement. Avec La Pensée 68, Ferry et Renaut s'attaquent aux prétendus successeurs de Sartre, les Foucault, Deleuze, Lyotard et Derrida qui, contrairement à Sartre, font valoir l'antihumanisme. Ferry et Renaut réévaluent l'humanisme et la raison moderne, se portent à la défense des droits de l'homme et à la restauration de l'idée républicaine. Avec Michel Henry, Paul Ricoeur et Emmanuel Levinas, on assiste à une inflexion theologique du mouvement phénoménologique amorcée déjà par Sartre dans sa version athée. Mais, ces dernières années, alors que se développent à l'échelle mondiale la science et la technique avec leurs implications épistémologiques et éthiques, la philosophie doit réévaluer ses priorités. Pour l'auteur, il s'agit de "rendre à nouveau raison des choses et des événements afin d'obtenir les rudiments d'une nouvelle sagesse ".

Tous les auteurs s'accordent, selon le vou des directeurs de l'ouvrage, à montrer les changements, les remaniements philosophiques survenus depuis au moins les deux dernières décennies, soit les années 70,80 et le début des années 90 . On remarquera, par exemple, une plus grande transparence grâce à une meilleure diffusion des penseurs de l'Est, en raison des événements que l'on a connus ces demières années dans ces pays auparavant limités dans leur liberté d'expression. Bref, par son aspect panoramique, cette première section constitue une excellente référence à qui veut connaitre les principaux courants philosophiques contemporains en Europe.

La seconde section, intitulée "Coups de sonde" consiste en quelques études ayant une portée universelle comme le sont les études de $\mathrm{K}$. O. Apel et de Hersch, le premier questionnant la possibilité d'une éthique universaliste, le second discutant des droits de l'homme selon un point de vue philosophique. D'autres études se rattachent plus directement à l'ensemble de l'Europe, comme le sont les deux dernières études, celles de Hengelbrock et de Muglioni, qui jettent un regard critique sur l'enseignement de la philosophie. D'une part, Hengelbrock se demande si l'enseignement de la philosophie en Europe est périmé ou indispensable et considère, selon une approche néo-kantienne, que l'école doit donner accès à la raison philosophique dans le but de créer "une rationalité critique qui seule garantit l'autonomie de la personne et l'usage positif de la libertè ", valeurs qui malheureusement s'enfouissent sous l'autoritarisme religieux, le retour aux mythes et l'incompréhension qui "coexistent de façon étonnante avec l'érudition scientifique la plus poussée "(p. 683). Pour sa part, Muglioni se penche sur l'avenir de la philosophie en Europe et insiste pour dire qu'il n'y a pas une philosophie européenne pas plus qu'il n'y a une philosophie américaine ou une philosophie orientale. Pour lui, la philosophie n'appartient pas à un pays, une région ou une école, mème si on peut, par exemple, identifier son lieu de naissance, la Grèce antique. Il faut relever aussi que l'auteur a une conception de l'enseignement de la philosophie bien différente des tendances actuelles favorisant la progressivité de l'enseignement de la discipline. Il serait vain de proposer un enseignement de la philosophie avant la classe terminale du secondaire, ce serait du saupoudrage, dit-il. On pourrait résumer la pensée de l'auteur en disant que l'avenir de l'Europe est redevable de sa conception de l'homme qui repose trop souvent sur des fictions passagères liées aux modes du présent, alors que la grandeur de l'homme réside dans sa capacité à réfléchir sur l'homme qui, comme il le reprend de Rousseau, "n'est pas ce que j'ai 
devant les yeux. le pédant, le courtisan du siècle, mais il vient de plus loin et ce qu'il parait résulte d'une histoire qui a recouvert sa nature au point de la rendre méconnaissable, comme la statue de Glaucus " (p. 807).

René O. Girard

Département de philosophie UQAM 